\title{
Adaptive Multipath Selection-Based Markov Chain in the Heterogeneous Internet of Things
}

\author{
Huan $W u,{ }^{1,2}$ Xiangming Wen $\mathbb{D}^{1},{ }^{1}$ Zhaoming Lu $\mathbb{D},{ }^{1}$ Yao Nie $\mathbb{D}^{2},{ }^{2}$ and Shuyang Huang ${ }^{1}$ \\ ${ }^{1}$ Beijing Key Laboratory of Network System Architecture and Convergence, \\ Beijing Laboratory of Advanced Information Networks, Beijing University of Posts and Telecommunications, \\ Beijing 100876, China \\ ${ }^{2}$ Anhui Engineering Research Center of Big Data of Specific Plant Resources, School of Electronics and Information Engineering, \\ West Anhui University, Lu'an 237012, China
}

Correspondence should be addressed to Xiangming Wen; xiangmw@bupt.edu.cn and Yao Nie; nieyao@wxc.edu.cn

Received 18 April 2019; Revised 30 July 2019; Accepted 30 August 2019; Published 15 November 2019

Academic Editor: Telmo Reis Cunha

Copyright (c) 2019 Huan Wu et al. This is an open access article distributed under the Creative Commons Attribution License, which permits unrestricted use, distribution, and reproduction in any medium, provided the original work is properly cited.

The Internet of Things (IoT) is a new heterogeneous system integrated by the various end users (sensors and terminals) with different technologies. However, the limiting factor is bandwidth in the IoT due to the exploding end users and the network bandwidth requirements. A novel IoT model, which integrates the power-line carrier (PLC) and the wireless network (WN), is proposed to solve the bandwidth problem from the architecture, especially in the areas lacking network facilities. In addition, we exploit an effective virtual layer (EVL) which allows the different end users to access the system model seamlessly. Then, the attractor selection algorithm based on Markov chain (MASA) is employed to select an optimal path among the PLC or WN. The simulation results demonstrate that the proposed system model has the smaller average queuing delay than other algorithms and makes the model more stable and robust.

\section{Introduction}

With the increasing requirements of the network services on account of the popularity of the smart end users (sensors and terminals), users expect the network environment in which they can readily access the internet at any time and any place. Especially in the Internet of Things (IoT), the smart end users are expected to reach 212 billion entities deployed globally at the end of 2020 [1], in which the machine-tomachine (M2M) traffic flows are expected to continue to grow up and the connections grow from 7.6 billion in 2015 to 8.0 billion in 2022 [2]. Thus, the IoT will contain the incredibly high number of the end users and be able to interconnect billions or trillions of the heterogeneous end users through the Internet, which leads to a critical requirement for the compatible network architecture. Moreover, the explosive data in the IoT lead to the bandwidth congestion and the lack of the spectrum resources. Therefore, accommodating more users, meeting the various demands of the users, connecting the end users seamlessly, and allocating the appropriate network resources dynamically are the inevitable requirements of the IoT.

In the traditional heterogeneous networks, the researchers mainly apply ultradense networks (UDN) to increase the network capacity [3]. That is to say, more base stations are deployed in the networks, which is a form of the superdense heterogeneous network. By far, the main structures of the heterogeneous wireless network (WN) are wireless local area networks- (WLAN-) worldwide interoperability for microwave access (WiMax), WiMaxWiFi, WLAN-universal mobile telecommunications system (UMTS), WiMax-3G (3rd generation), etc. [4-6]. Simultaneously, the spectral efficiency of the wireless communication approximates to Shannon limit based on the traditional orthogonal frequency division multiplexing (OFDM) and the multiple input multiple output (MIMO). However, these structures cannot completely solve the problem of the network capacity due to the 
limited wireless spectrum resources, while the network capacity requirements grow with the number of the end users increased. In addition, to provide more coverage requires more base stations deployed and more constructions and maintenance costs. Fortunately, there is a novel communication technology, which uses the power line to transmit information, named "power-line communication" (PLC). PLC is a kind of high-speed information transmission, which is applied in the smart metering and smart grid widely to improve the degree of smart grid and smart city [7, 8]. In addition, PLC has obvious advantages in the wider coverage areas, such as stability and reliability, and without any need for the additional communication lines. These advantages are important and cost-effective for the users in the IoT to coverage the blind areas and hotspots, such as, the countryside, mountain area, no-human-zone, and commercial hotspots or community hotspots. Therefore, we propose a novel system model which integrates the PLC and WN for the IoT named the "heterogeneous IoT" (HIoT). The HIoT is used to realize the effective utilization of complementary characteristics of the different networks.

In the traditional networks, there are three main types of selection path algorithms: the path selection based on multiple attribute decision making (MADM), the path selection based on fuzzy logic, and the path selection based on game theory. The path selection based on MADM includes compensatory algorithm and noncompensatory algorithm. The noncompensatory algorithm is used to find the candidate network which meets the minimum requirement of the terminal, while the compensatory algorithm chooses the optimal network by considering multiple attributes. The most MADM algorithms are the compensatory algorithm in the heterogeneous network. Wu et al. [9] combine Received Signal Strength (RRS) trigger threshold values and the AHP-derived weights and score functions to decide the best candidate network which avoids frequent handover in the WiFi and WiMAX networks. And Hwang et al. [10] consider the effects of SINR, user required bandwidth, user traffic cost, and the available bandwidth of the participating access networks as the important parameters to select the target network in the heterogeneous WN. The fuzzy logic is the core algorithm for the network selection algorithms. Considering the drone moving and communicating in 3-D space, the paper [11] applies a fuzzy inference method for the handover decision. This fuzzy inference uses the speed limit and coverage as input variables. Thus, the fuzzy logic algorithm in [11] is suitable for communication environments of drones and is better than the traditional method. In [12], the fuzzy logic using the quantitative decision algorithm makes a final decision to select the target network with the largest quantitative decision value (QDV). In the results, the proposed algorithm outperforms other algorithms. The third method named game theory [13] is widely used in many fields, such as in computer networks. Malanchini et al. [14] propose a noncooperative game theory to model the competition among the multiple end users in accessing shared wireless networks. And the simulation results show the proposed game model in [14] is suitably adopted to represent and drive the dynamics of the practical network scenarios. In [15], the authors apply the noncooperative dynamic game with the end devices for the game players and the IoT gateways for the selection strategy. In the simulation results, the total demands for the end devices and the load balance among the gateways are efficient than in the other previous studies. Up to date, no solutions have been proposed for the integration of the PLC and WN in the IoT. Therefore, we propose the research contributions are required.

In this paper, we propose the heterogeneous PLC and WN model for the IoT, which is the extended work based on our previous work [16]. Different from the two-path model in our previous work, the multipath model for the realistic network scenario is proposed. Based on the multipath model, the transfer probability and the numeric results of the average queuing delay are also different from that under the two-path model in [16]. In addition, the multiuser scenario is investigated. Thus, the path selection algorithm based on the multipath Markov chain is extended based on the two-path Markov chain in [16]. Then, we add the compared algorithms, which are simple additive weighting (SAW) and interference-suppression resource optimization based on potential game (ISRO_PG), in the simulation. Furthermore, we add the comparison of the average queuing delay and the ratio of the different algorithms with the number of different nodes, which are not discussed in [16], to verify the performance of the proposed algorithm in the simulation results. The simulation results show that the algorithm in [16] is not suitable for the multipath scenario with the increased data. And the proposed algorithm makes the network scalability (the number of nodes can be very high), reliability (the network may report urgent alarm events), and robustness (transmission nodes are likely to be subject to failures for several reasons).

The rest of this paper is organized as follows. The system model is surveyed in Section 2. We show the mathematical model of the queuing and the attractor selection algorithm in Section 3. This is followed by a description of how to apply the attractor selection algorithm in the HIoT in Section 4. And, we evaluate the performances by some exemplary simulation results which are presented in Section 5. Finally, this paper is concluded in Section 6.

\section{System Model}

In this section, we propose the system model of the HIoT. We define a virtual layer for the protocol conversion. Then, the queuing model, which is the foundation for the mathematical calculations, is provided. Finally, we present the control model which ensures the normal operations of the system model.

2.1. Virtual Layer. Many access technologies, including cellular, WiFi, WiMAX, PLC, Long-Term Evolution (LTE), and General Packet Radio Service (GPRS) coexist in IoT. The protocols and the formats of the packets (the frames) are different from those of the different access technologies $[17,18]$. There is thus the need for an abstraction layer to 
harmonize the access of the different devices and technologies with a common language and procedure. We assume the abstraction layer named "effective virtual layer" (EVL) as shown in Figure 1, which is a virtual layer interposed between the data link layer and the physical layer. Its feature is to hide the details of the different access technologies for the upper applications. The EVL consists of two main sublayers: the interface sublayer and the communication sublayer. The first sublayer provides the compatible interface which allows the various technologies to access the HIoT. The second sublayer is a wrapping layer, which makes the protocols and the formats of the packets into the standard service language.

As shown in Figure 1, the identity information of the data is added into the information head on each layer as the following step. Firstly, the port identity document (ID) is added into the information head of the data while the data are transmitted to the transport layer of the end-to-end transmission. Then, the data are delivered to the network layer and added with the internet protocol (IP) address of the source, the destination, and the routing protocol number of the network routing into the information head. Finally, the media access control address (MAC) is added into the information head, while the data are transmitted downward to the data link layer. Therefore, it is can be seen that the data are repackaged in EVL to hide the varied transmission media, which are different from the traditional transmission process. This process is very important and is the premise of the whole paper. Based on the above process, we assume that the nodes can access the HIoT through the PLC or WN for the ubiquitous and seamless connectivity.

2.2. Queuing Model. While the data (e.g., files and packets) arrive in the node (sensor) to find another data currently being queued or in transmission, the data stand in the queue. The queuing model of the HIoT is shown in Figure 2. The queue has two states (the PLC or the WiFi), and the queue process obeys server vacations and impatient customers or with reneging [19-21]. At the same time, the data belonging to the same type obey the first come first served (FCFS) queuing discipline.

2.3. Access Control Mechanism. We assume that every node has the access control mechanism to coordinate the transmission process. The access control mechanism consists of four parts: the feedback module, the storage module, the algorithm module, and the scheduling module. The feedback module collects the path information (congestion, disconnection, etc.) between the two nodes which communicate with each other. The information is transmitted to the algorithm module based on MASA for selection of the optimal path. The algorithm module is the core in the access control mechanism, and the algorithm module progress is shown in Figure 3. We can see that the node continues to transmit the data by the current path (CP) while the CP is in the good condition. In contrast, while the node's $\mathrm{CP}$ is not suitable for transmitting the data, the node selects the optimal transmission path based on the path selection algorithm (MASA). The storage module is used to store the important information, for instance, the path information. The scheduling module is mainly used for the path adjustment and the switching process. When the data come into the node, the scheduling module puts the data into a suitable queue based on MASA.

\section{Queuing Model for the System}

According to the queuing theory, the mean queue size is one of the important indicators to judge the system performance so that it is widely used in the network research studies [22-24]. Also, in our system, the proposed algorithm based on the average queuing delay does not require the powerful processing capacity of the nodes leading to that the terminals with the different performances can access the HIoT. Thus, a mass of terminals can easily access the network with low cost in the future, especially in the IoT or the smart home. Moreover, the average queuing delay can effectively avoid the impact of the unexpected conditions, such as the unnecessary network (path) selection impacting the network performance as the PingPong effect in the cellular network. Therefore, we exploit the average queuing delay as the criterion in this paper. In this paper, the queuing process can be modeled with the transition graph of the two-path Markov chain. Then, the average queuing delay of different networks can be obtained. Considering the variety of the real networks, we extend the two-path Markov chain to multipath Markov chain. Finally, the average queuing delay of multipath Markov chain is obtained.

3.1. Average Queuing Delay in the Two-Path Markov Chain. For the convenience of research, the two-path Markov chain is applied to the queuing process between the PLC and WiFi which models the simple HIoT. The two-path Markov chain for the HIoT model is illustrated in Figure 4. In the HIoT, the two-path Markov chain has two states, the PLC state and the WiFi state. In the PLC state, the arrival and service rates are $\lambda_{1}$ and $\mu_{1}$, respectively. And the PLC state tends "to jump" to the WiFi state with Poisson intensity $\eta_{1}$. Similarly, the arrival and the service rates of the WiFi are $\lambda_{2}$ and $\mu_{2}$, respectively. Also, the WiFi state tends "to jump" to the PLC state with Poisson intensity $\gamma_{1}$. The useful notations are depicted in Table 1.

According to the transfer characteristic of the Markov chain, the average rate at which a point (state) is entered equals to the average rate at which a transition from the point occurs. Thus, the equilibrium state in Figure 4 can be written as

$$
\begin{gathered}
P_{0, P}\left(\lambda_{1}+\eta_{1}\right)=\mu_{1} P_{1, P}+\gamma_{1} P_{0, W}, \\
P_{0, W}\left(\lambda_{2}+\gamma_{1}\right)=\mu_{2} P_{1, W}+\eta_{1} P_{0, P},
\end{gathered}
$$




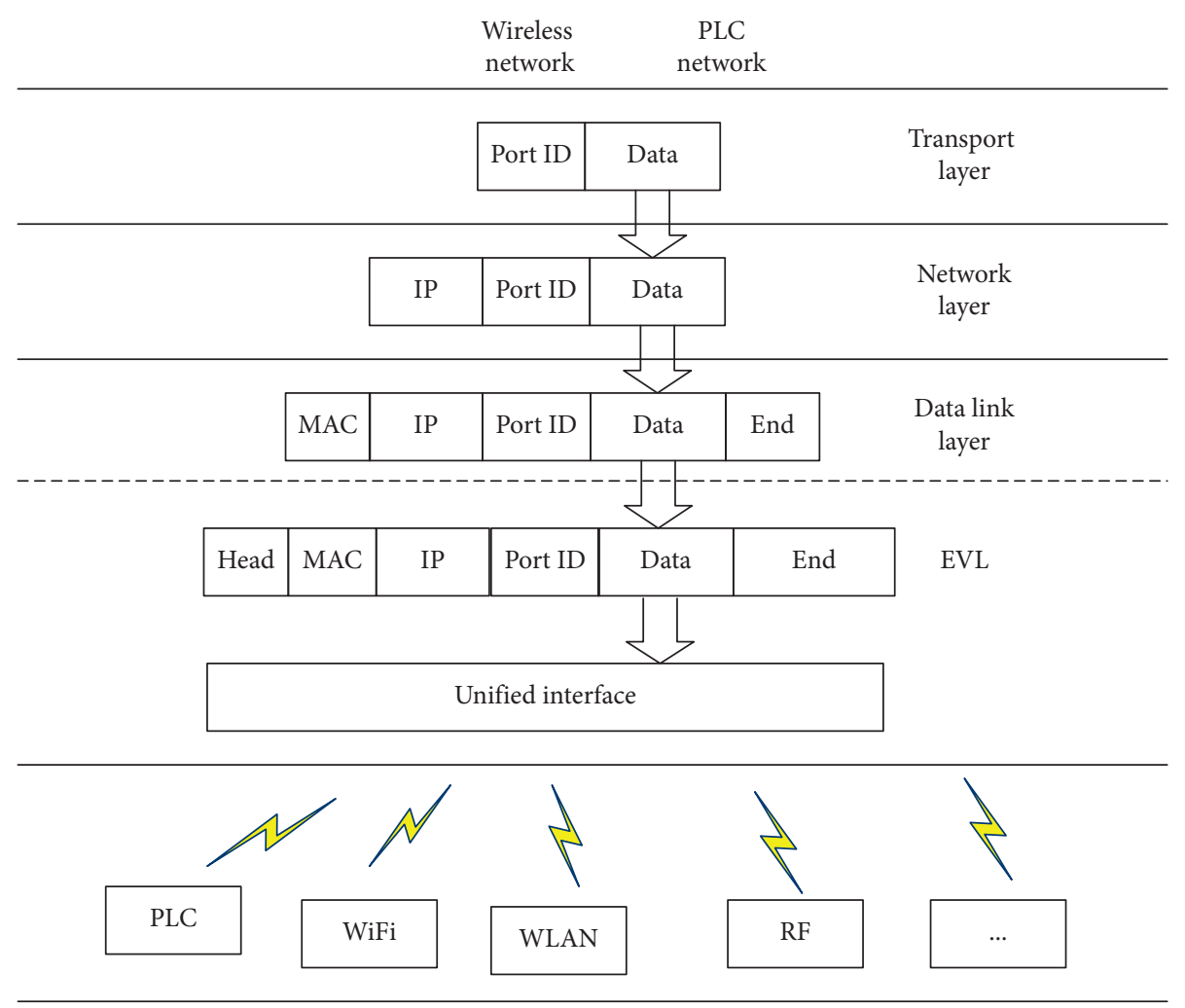

Figure 1: Process of the protocol conversion.

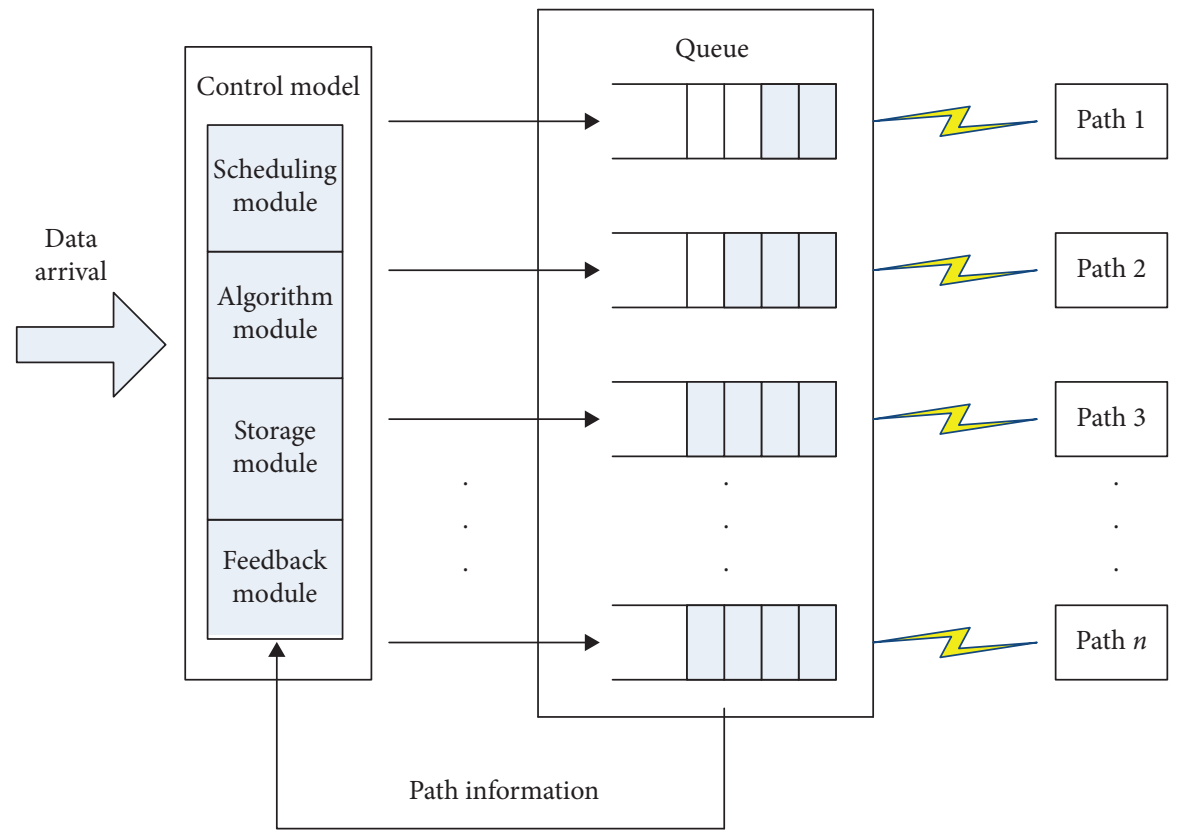

Figure 2: Queuing model of the HIoT.

$$
\begin{gathered}
P_{n, P}\left(\lambda_{1}+\eta_{1}+\mu_{1}\right)=\lambda_{1} P_{n-1, P}+\mu_{1} P_{n+1, P}+\gamma_{1} P_{n, W}, \\
P_{n, W}\left(\lambda_{2}+\gamma_{1}+\mu_{2}\right)=\lambda_{2} P_{n-1, W}+\mu_{2} P_{n+1, W}+\eta_{1} P_{n, P} .
\end{gathered}
$$

The partial generating functions of the model are defined as

$$
\begin{aligned}
G_{P}(z)=\sum_{n=0}^{\infty} z^{n} P_{n, P}, & |z| \leq 1, \\
G_{W}(z)=\sum_{n=0}^{\infty} z^{n} P_{n, W}, & |z| \leq 1 .
\end{aligned}
$$




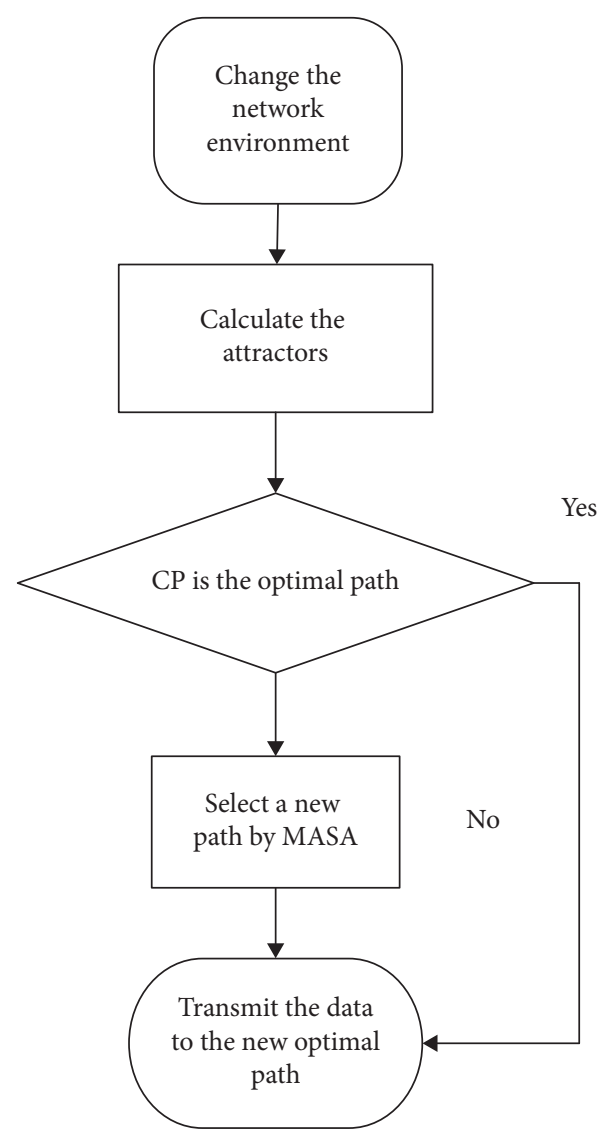

Figure 3: Algorithm module process.

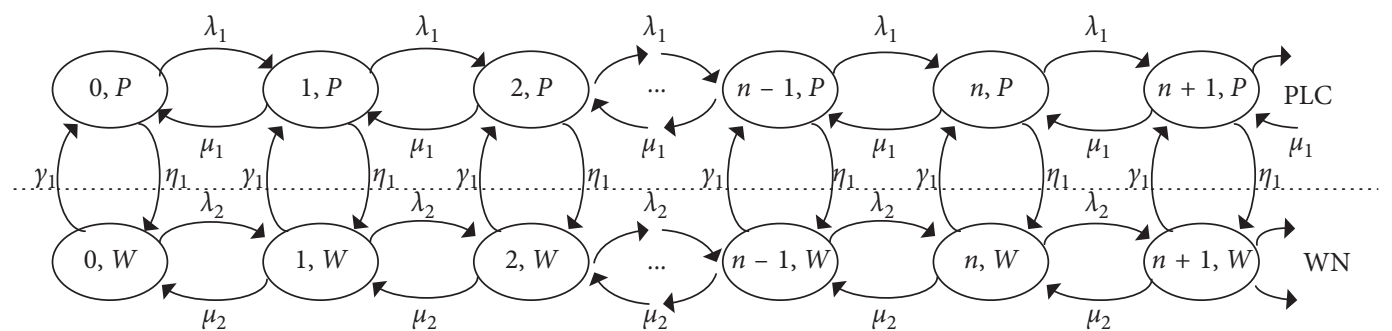

Figure 4: Two-path Markov chain for the HIoT queuing.

TAвLe 1: Variables and definition.

\begin{tabular}{lr}
\hline Variable & Definition/description \\
\hline$\lambda_{1} / \lambda_{2}$ & The arrival rate of the PLC or the WiFi \\
$\mu_{1} / \mu_{2}$ & The service rate of the PLC or the WiFi \\
$P_{n, P}$ & The probability of finding $n$ files in the PLC state \\
$P_{n, W}$ & The probability of finding $n$ files in the WiFi state \\
$P_{P}$ & The probability of the system being at the PLC state \\
$P_{W}$ & The probability of the system being at the WiFi state \\
$\eta_{1}$ & The rate of leaving the PLC state \\
$\gamma_{1}$ & The rate of leaving the WiFi state \\
\hline
\end{tabular}

Then, (1), (2), (3), and (4) are multiplied by $z^{n}(n=$ $0,1, \ldots)$ appropriately and added over all $n$. These processing results are

$$
\begin{aligned}
\left(\lambda_{1}+\eta_{1}+\mu_{1}\right) G_{P}(z)= & \lambda_{1} z G_{P}(z)+\gamma_{1} G_{W}(z) \\
& +\frac{\mu_{1}}{z}\left[G_{P}(z)-P_{0, P}\right]+\mu_{1} P_{0, P}, \\
\left(\lambda_{2}+\gamma_{1}+\mu_{2}\right) G_{W}(z)= & \lambda_{2} z G_{W}(z)+\eta_{1} G_{P}(z) \\
& +\frac{\mu_{2}}{z}\left[G_{W}(z)-P_{0, W}\right]+\mu_{2} P_{0, W} .
\end{aligned}
$$

According to (6) and (7), we get

$$
\begin{aligned}
f(z) G_{P}(z)= & P_{0, P} \mu_{1}\left[\gamma_{1} z+\lambda_{2} z(1-z)-\mu_{2}(1-z)\right] \\
& +\gamma_{1} \mu_{2} P_{0, W} z,
\end{aligned}
$$


where

$$
\begin{aligned}
f(z)= & \lambda_{1} \lambda_{2} z^{3}-\left(\eta_{1} \lambda_{2}+\gamma_{1} \lambda_{1}+\lambda_{1} \lambda_{2}+\lambda_{1} \mu_{2}+\lambda_{2} \mu_{1}\right) z^{2} \\
& +\left(\eta_{1} \mu_{2}+\gamma_{1} \mu_{1}+\mu_{1} \mu_{2}+\lambda_{1} \mu_{2}+\lambda_{2} \mu_{1}\right) z-\mu_{1} \mu_{2}
\end{aligned}
$$

From (8), we do need to determine the probabilities of $P_{0, W}$ and $P_{0, P}$. It can be proven that the polynomial in (9) has only one root in the open interval $(0,1)$ [25]. This root is denoted as $z_{0}$ into (8), so we have

$$
P_{0, P} \mu_{1}\left[\gamma_{1} z_{0}+\lambda_{2} z_{0}\left(1-z_{0}\right)-\mu_{2}\left(1-z_{0}\right)\right]+P_{0, W} \gamma_{1} \mu_{2} z_{0}=0
$$

Since $P_{0, W}$ and $P_{0, P}$ are unknown, we need another condition to obtain the zero probabilities. Writing the balance equation through a vertical cut between states containing $n$ and $n+1$ files, we obtain

$$
\mu_{1} P_{n+1, P}+\mu_{2} P_{n+1, W}=\lambda_{1} P_{n, P}+\lambda_{2} P_{n, W} .
$$

Summation of (11) over all $n$ yields

$$
\lambda_{1} P_{P}+\lambda_{2} P_{W}=\mu_{1}\left(P_{P}-P_{0, P}\right)+\mu_{2}\left(P_{W}-P_{0, W}\right),
$$

where $P_{P}=\sum_{n=0}^{\infty} P_{n, P}$, which means the probability of the system being at the PLC state only, and $P_{W}=\sum_{n=0}^{\infty} P_{n, W}$, which means the probability of the system being at the WiFi state only. Then, using standard renewal theory [26], we have $P_{W}=\eta_{1} /\left(\eta_{1}+\gamma_{1}\right)$ and $P_{P}=\gamma_{1} /\left(\eta_{1}+\gamma_{1}\right)$. Solving the equation set consisting of (11) and (12), we have the 0 th state probability of the PLC and the WiFi as

$$
\begin{gathered}
P_{0, P}=\frac{\gamma_{1}(\mu-\lambda) z_{0}}{\mu_{1}\left(1-z_{0}\right)\left(\mu_{2}-\lambda_{2} z_{0}\right)}, \\
P_{0, W}=\frac{\eta_{1}(\mu-\lambda) z_{0}}{\mu_{2}\left(1-z_{0}\right)\left(\mu_{1}-\lambda_{1} z_{0}\right)} .
\end{gathered}
$$

Finally, from (6) and (7), $G_{P}(z)$ and $G_{W}(z)$ are given by

$$
\begin{aligned}
G_{P}(z) & =\frac{\gamma_{1}(\mu-\lambda) z+P_{0, P} \mu_{1}(1-z)\left(\lambda_{2} z-\mu_{2}\right)}{f(z)}, \\
G_{W}(z) & =\frac{\eta_{1}(\mu-\lambda) z+P_{0, W} \mu_{2}(1-z)\left(\lambda_{1} z-\mu_{1}\right)}{f(z)} .
\end{aligned}
$$

We define auxiliary quantities $N_{P}=\sum_{n=0}^{\infty} n P_{n, P}$ and $N_{W}=\sum_{n=0}^{\infty} n P_{n, W}$. Clearly, we get $N_{P}=\left.(\mathrm{d} / \mathrm{d} z) G_{P}(z)\right|_{z=1}$ and $N_{W}=\left.(\mathrm{d} / \mathrm{d} z) G_{W}(z)\right|_{z=1}$. to be

Then, after some development, $N_{P}$ and $N_{W}$ are found

$$
\begin{gathered}
N_{P}=\frac{\gamma_{1}(\mu-\lambda)+P_{0, P} \mu_{1}\left(\mu_{2}-\lambda_{2}\right)}{S}-\frac{\gamma_{1}(\mu-\lambda) R}{S^{2}}, \\
N_{W}=\frac{\eta_{1}(\mu-\lambda)+P_{0, W} \mu_{2}\left(\mu_{1}-\lambda_{1}\right)}{S}-\frac{\eta_{1}(\mu-\lambda) R}{S^{2}},
\end{gathered}
$$

where

$$
\begin{aligned}
S= & \eta_{1}\left(\mu_{2}-\lambda_{2}\right)+\gamma_{1}\left(\mu_{1}-\lambda_{1}\right), \\
R= & \lambda_{1} \lambda_{2}-\left(\eta_{1}+\mu_{1}\right) \lambda_{2}-\left(\gamma_{1}+\mu_{2}\right) \lambda_{1}+\eta_{1}\left(\mu_{2}-\lambda_{2}\right) \\
& +\gamma_{1}\left(\mu_{1}-\lambda_{1}\right) .
\end{aligned}
$$

The quantities $N_{P}$ and $N_{W}$ can be considered as the mean queue size in the PLC state and the WiFi state, respectively. Finally, using Little's law $E[N]=\lambda E[T]$ [27], the average queuing delay in the PLC state and the WiFi state are $E\left[T_{P}\right]=N_{P} / \lambda_{1}$ and $E\left[T_{W}\right]=N_{W} / \lambda_{2}$, respectively.

3.2. Average Queuing Delay in the Multipath Markov chain. In the previous work [16], we considered a simple HIoT scenario with two networks (PLC and WiFi). Nevertheless, many access technologies coexist, such as LTE, GPRS, WiFi, and PLC which cover the same areas in the HIoT. In the real scenarios, the end users may be switched between some different technologies and/or rates, in which the end users need different access technology support. Therefore, twopath Markov chain in the HIoT is extended to the multipath Markov chain to be suitable for the multipath queuing model which represents the multiple paths.

Based on the above assumptions, the multipath Markov chain is shown in Figure 5. We assume that each level (row) in this figure corresponds to a transmission path, which can be exploited using the same or different technology. Note that the scenario, which has a number of different rates and the users could experience within the same technology, can also be applied by this Markov chain. The stationary probability of state $\{j, i\}$, denoted $P_{j, i}$, which represents $j$ files in the buffer at the $i$ th path. The data arrive in path $i$ according to a homogeneous Poisson process with intensity $\lambda_{i}$; for example, at $P_{j, i}$, the chain moves to the right state (horizontally) with rate $\lambda_{i}$. Service times at $i$ th path are assumed to be independent and identically distributed with a density $\mu_{i}$. Similarly, the chain moves to the left (horizontally) with rate $\mu_{i}$. The chain moves (vertically) from the $i$ th level to another $\bar{i}$ th level with rate $\eta_{i, \bar{i}}$, where $\bar{i}=1,2$, $3, \ldots, M, \bar{i} \neq i$. Here, $\eta_{i}=\sum_{\bar{i}=1}^{M} \eta_{i, \bar{i}}$, which means all possible transition changes in the network connectivity at $i$ th level. Based on the above assumptions, the balance equations for the multipath Markov chain can be written as

$$
\left(\lambda_{i}+\eta_{i}\right) P_{0, i}=\mu_{i} P_{1, i}+\sum_{\bar{i}=1}^{M} \eta_{\bar{i}, i} P_{0, \bar{i}}, \quad j=0, i=1,2,3, \ldots, M,
$$

$$
\begin{array}{r}
\left(\lambda_{i}+\mu_{i}+\eta_{i}\right) P_{j, i}=\lambda_{i} P_{j-1, i}+\mu_{i} P_{j+1, i}+\sum_{\bar{i}=1}^{M} \eta_{\bar{i}, i} P_{j, \bar{i}} \\
j>0, i=1,2,3, \ldots, M .
\end{array}
$$

Then, we derive the expression of the average queuing delay in the HIoT system. Let the generating functions for each path be 


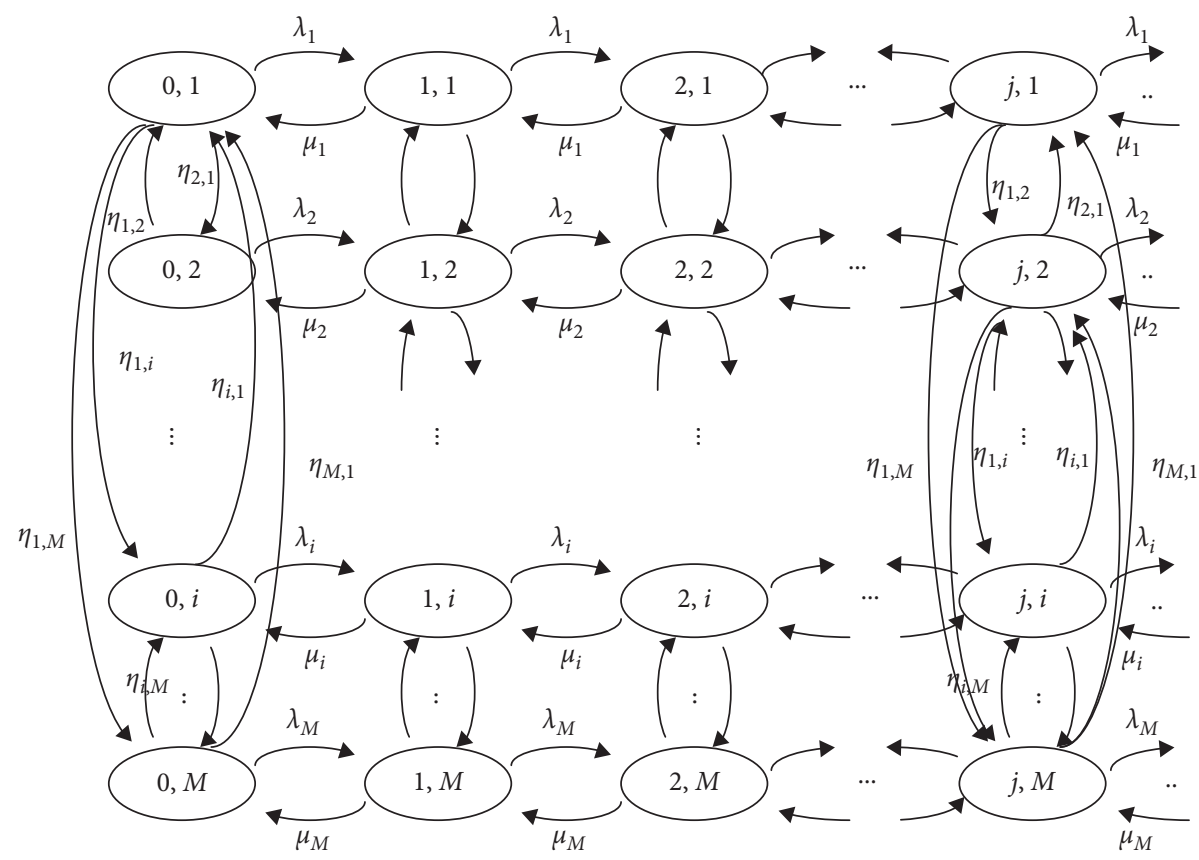

FIGURE 5: Multipath Markov chain for the HIoT queuing.

$$
G_{i}(z)=\sum_{j=0}^{\infty} z^{j} P_{j, i}, \quad|z \leq 1|, i=0,1,2, \ldots, M
$$

Summing (17) and (18), and multiplying by $z^{j}$, we get

$$
\begin{aligned}
\lambda_{i} \sum_{j=0}^{\infty} P_{j, i} z^{j}+\mu_{i} \sum_{j=1}^{\infty} P_{j, i} z^{j}+\eta_{i} \sum_{j=0}^{\infty} P_{j, i} z^{j}= & \lambda_{i} \sum_{j=1}^{\infty} P_{j-1, i} z^{j} \\
& +\mu_{i} \sum_{j=0}^{\infty} P_{j+1, i} z^{j} \\
& +\sum_{j=0}^{\infty} z^{j} \sum_{\bar{i}=1}^{M} P_{j, i} \eta_{\bar{i}, i} .
\end{aligned}
$$

After introducing the substitution, (20) can be rewritten as

$$
\begin{aligned}
\lambda_{i} G_{i}(z)+\mu_{i}\left[G_{i}(z)-P_{0, i}\right]+G_{i}(z) \eta_{i}= & \lambda_{i} z G_{i}(z) \\
& +\frac{\mu_{i}}{z}\left[G_{i}(z)-P_{0, i}\right] \\
& +\sum_{\bar{i}=1}^{M} \eta_{\bar{i}, i} G_{\bar{i}}(z) .
\end{aligned}
$$

After performing some algebra, we have

$$
f_{i}(z) G_{i}(z)-\sum_{\bar{i}=1}^{M} \eta_{\bar{i}, i} z G_{\bar{i}}(z)=\mu_{i}(z-1) P_{0, i}, \quad i=1,2, \ldots,
$$

where $f_{i}(z)=\lambda_{i} z(1-z)-\mu_{i}(1-z)+\eta_{i} z$.
Then, we put (22) into the following matrix:

$$
\mathbf{A}(z) g(z)=(z-1) \boldsymbol{\theta}
$$

where

$$
\begin{aligned}
\mathbf{A}(z) & =\left[\begin{array}{ccccc}
f_{1}(z) & -\eta_{2,1} z & -\eta_{3,1} z & \cdots & -\eta_{M, 1} z \\
-\eta_{1,2} z & f_{2}(z) & -\eta_{3,2} z & \cdots & -\eta_{M, 2} z \\
\vdots & \vdots & \vdots & \ddots & \vdots \\
-\eta_{1, M} z & -\eta_{2, M} z & -\eta_{3, M} z & \cdots & f_{M}(z)
\end{array}\right], \\
g(z) & =\left[\begin{array}{c}
G_{1}(z) \\
G_{2}(z) \\
\vdots \\
G_{M}(z)
\end{array}\right], \\
\boldsymbol{\theta} & =\left[\begin{array}{c}
\mu_{1} P_{0,1} \\
\mu_{2} P_{0,2} \\
\vdots \\
\mu_{M} P_{0, M}
\end{array}\right] .
\end{aligned}
$$

By applying Cramer's rule to (23), we can obtain

$$
G_{i}(z)=\frac{\left|\mathbf{A}_{i}(z)\right|(z-1)}{|\mathbf{A}(z)|}, \quad i=0,1,2, \ldots, M,
$$

where $|\mathbf{A}(z)|$ stands for the determinant of matrix $\mathbf{A}(z)$ and the matrix $\mathbf{A}_{i}(z)$ is obtained by replacing the $M$ th column of $\mathbf{A}(z)$ with $\boldsymbol{\theta}$. Note that the denominator $|\mathbf{A}(z)| / z-1$ is a polynomial of degree $2 M-1$. It has been pointed out in [28] that at $z=1,|\mathbf{A}(z)|=0$ and $|\mathbf{A}(z)|=(z-1) Q(z)$. Here, $\mathrm{Q}(z)$ is some polynomial of degree $2 M$. Thus, (25) can rewritten as 


$$
Q(z) G_{i}(z)=\left|\mathbf{A}_{i}(z)\right|, \quad i=0,1, \ldots, M
$$

The polynomial $Q(z)$ has exactly $M-1$ distinct real roots in the interval $(0,1)$ [25]. Let $z=z_{m}, m=1,2$, $\ldots, M-1$, be the $M-1$ distinct roots of $Q(z)$. In (26), we obtain

$\left|\mathbf{A}_{i}\left(z_{m}\right)\right|=0,(m=1,2, \ldots, M-1 ; i=0,1,2, \ldots M)$. For each $z_{m}$, we have $M+1$ homogeneous linear equations in the unknown probabilities $P_{0,1}, P_{0,2}, \ldots, P_{0, M}$. Let us consider the vertical cut between states $j$ and $j+1$. The balance equation through the cut is

$$
\lambda_{1} P_{j, 1}+\lambda_{2} P_{j, 2}+\cdots+\lambda_{M} P_{j, M}=\mu_{1} P_{j+1,1}+\cdots+\mu_{M} P_{j+1, M} .
$$

Summation of (27) over all $j$ yields

$\lambda_{1} P_{1}+\lambda_{2} P_{2}+\cdots+\lambda_{M} P_{M}=\mu_{1}\left(P_{1}-P_{0,1}\right)+\cdots+\mu_{M}\left(P_{M}-P_{0, M}\right)$,

where $P_{i}=\sum_{j=0}^{\infty} P_{j, i}$ denotes the percentage of time of the system in the ith path. Then, (28) can be rewritten as

$$
\widehat{\mu}-\widehat{\lambda}=\sum_{i=1}^{M} \mu_{i} P_{0, i}
$$

where $\widehat{\mu}=\sum_{i=1}^{M} \mu_{i} P_{i}$ is the average capacity of the system to render service and $\hat{\lambda}=\sum_{i=1}^{M} \lambda_{i} P_{i}$ is the average rate of customer arrivals. Thus, the probabilities $P_{i}$ need to be determined firstly. We define $q_{i, \bar{i}}=\eta_{i, \bar{i}} / \eta_{i}$, which is the transition probabilities. According to [25], we have

$$
P_{i}=\frac{d_{i} / \eta_{i}}{\sum_{i=1}^{M} d_{i} / \eta_{i}}
$$

where $d_{i}$ is the solutions to the global balance equations. So, $\sum_{i=1}^{M} d_{i}=1$, and $d_{\bar{i}}=\sum_{i=1}^{M} d_{i} q_{i, \bar{i}}$.

Replacing (30) into (29), we have the $M$ th equations of our system. Now, by solving this system, we get all the zero probabilities. From (26), the partial PGFs are found as

$$
G_{i}(z)=\frac{\left|\mathbf{A}_{\mathbf{i}}(z)\right|}{\mathrm{Q}(z)}, \quad i=1, \ldots, M
$$

The average number of files in the system is

$$
E[N]=\sum_{i=1}^{M} G_{i}^{\prime}(1)
$$

Using Little's law $E[N]=\lambda_{i} E[T]$, the average queuing delay in the multipath HIoT is given by

$$
E\left[T_{i}\right]=\left.\frac{1}{\lambda_{i}} \sum_{i=1}^{M}\left(\frac{\left|\mathbf{A}_{i}(z)\right|}{Q(z)}\right)^{\prime}\right|_{z=1} .
$$

\section{Attractor Selection Algorithm}

4.1. Extend Attractor Selection Algorithm. The original attractor selection algorithm (OASA), which has two attractors corresponding to the two proteins, can adaptively change the external form of the cell according to the external environment. The OASA was applied in the network research studies in
[29-31] because of the similarity between the access network selection adapted to the network environment and the cells adapted to the external environment. Therefore, the network configuration, which represents the operators in the OASA, achieves the purpose of selecting the optimal network path by controlling the operator selection. The papers [32-34] map the variables of the network, which are network performance parameters including the network time delay, throughput, and packet loss rate to the attractors. And then, the network can adjust the allocation of the resources according to the environment. However, the OASA is limited to the two attractors which are not applicable to the dynamic HIoT environment with the multipath. Thus, we extend the OASA named "EASA" to the multipath which has $M$ th attractors as follows:

$$
\frac{\mathrm{d} m_{i}}{\mathrm{~d} t}=\frac{S(A)}{1+m_{C P}^{2}-m_{i}^{2}}-D(A) \times m_{i}^{2}+\varepsilon_{i}, \quad i=1,2, \ldots, M,
$$

where $m_{i}$ is the value of path $i$, which represents the transmission performance of the path $i . m_{\mathrm{CP}}$ is the value of CP. $A$ is the activity. In the multipath selection investigation, we can map the network environment to $A$. Moreover, $S(A)$ and $D(A)$ are the monotonously increasing nonnegative function of $A$. $\varepsilon_{i}$ denotes the internal noises in the gene expression, which are independent of each other.

For the sake of simplicity, we denote $\Phi(A)$ as follows: $\Phi(A)=S(A) / D(A)$, without considering the noise terms; then, the following equation can be obtained from (34):

$$
m_{i}= \begin{cases}\Phi(A), & \text { high value, } \\ \frac{1}{2}\left[\sqrt{4+\Phi(A)^{2}}-\Phi(A)\right], & \text { low value. }\end{cases}
$$

According to the selection attractor of the biology, the selection process can be described as follows: The cell adapts to the environment, which means that $A$ is in the high value. And the cell converges to an attractor, and it is in a stable state under the high value of $A$ condition. While the environment changes, the cell maintains stable state and the attractor remains unchanged if the value of $A$ is still high. On the contrary, $S(A)$ and $D(A)$ decrease with decreases of the value of $A$ under the environment-changed condition. Then, the noise item of Equation (34) is in the dominant position. Thus, the expression of cell changes with the random noise, and the cell reselects the attractor. Until the value of $A$ increases, the new attractor is selected to restore the cell to the active state. $S(A)$ and $D(A)$ increase, and the noise items of Equation (34) can be ignored simultaneously.

In the HIoT, all transmission paths between the two nodes can be considered as the solution set. The value of activity $A$ is a measure of the adaptation of the path to the environment. From Equation (35), the solution set includes a solution with high value and many solutions with low value, which correspond to the optimal path and the other paths in the HIoT. The variation of the selection probabilities over the parameter $A$ is shown in Figure 6. We define $S(A)=$ $A\left[a A^{b}+1 / \sqrt{2}\right]$ and $D(A)=A$, where parameters $a$ and $b$ are the factors which can be set at $a=5$ and $b=2$ [35]. 


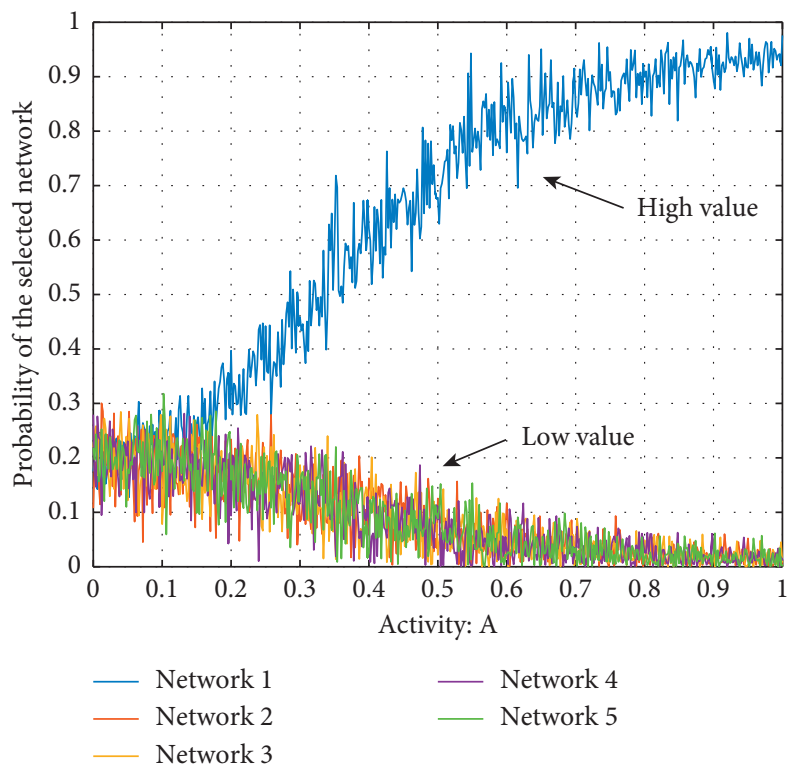

FIgURE 6: Variation of the selection probabilities over the parameter $A$.

Figure 7 depicts the multipath selection process, and it can be seen that the two nodes are connected by many paths. Usually, the source node $(S)$ transmits the data by the optimal path according to the path selection algorithm. However, the node executes the algorithm of scheduling to select the new path under the performance degradation or transmission failure condition which means that the $\mathrm{CP}$ is not suitable to transmit the data. That is to say, the environment is not suitable to transmit the data with the decrease of the value of $A$, and then, the node selects the new optimal transmission path according to Equation (35). In contrast, the node continues to transmit the data by the $\mathrm{CP}$ under the high value of $A$, which means that the system adapts to the environment.

4.2. Mapping A to the Network. In this section, we provide the activity expression in the HIoT. Based on the adaptive attractor selection of the biology, the cell expression can be regarded as the path state and the attractor can be regarded as the path in the network selection. The high value of $A$ means that the path adapts to the transmission. Thus, the system (the network system) converges to the optimal path and is under a stable state. Although the path state changes, the system maintains the stable state and the transmission path remains unchanged due to the high value of $A$. On the contrary, $S(A)$ and $D(A)$ decrease with the decreases of the value of $A$ under the path state-changed condition. Also, the noise item of Equation (34) is in the dominant position. Therefore, the path state changes with the random noise, and the system reselects the optimal path. In this procedure, the system exists a random state of change, if the new path is still unable to restore the value of $A$. Until the value of $A$ increases, the new optimal path restores the system to the active state. Meanwhile, $S(A)$ and $D(A)$ increase, the noise item of Equation (34) can be ignored and the system returns to the stable state. Thus, the goal of mapping $A$ to the network is to select the optimal path according to the average queuing delay of the different paths. That is to say, the HIoT exploits the EASA to select the path of the PLC or WiFi for transmitting data based on the shorter average queuing delay.

Therefore, we map the average queuing delay into $A$ which can represent the network environment (the queuing length or delay) as follows:

$$
A=1-\frac{1}{1+e^{-\kappa\left[T_{\mathrm{CP}}^{*}-\Delta\right]}},
$$

where $T_{\mathrm{CP}}^{*}$ is the normalized result of $T_{\mathrm{CP}}$, which means the average queuing delay of the $\mathrm{CP}$ in the HIoT. And $\kappa$ is a constant which influences the curves of the mapping activity. The relationship between $T_{\mathrm{CP}}^{*}$ and $T_{\mathrm{CP}}$ is shown in Figure 8 . From the figure, we can see that $T_{\mathrm{CP}}^{*}$ changes between 0 and 1 and its value increases with the value of $T_{\mathrm{CP}}$ increases.

Figure 9 shows the relationship between $A$ and $\kappa$. It can be found that the parameter $\kappa$ can be used to control the shape of $A$. The larger the parameter $\kappa$ is, the steeper the slope of $A$ becomes. $\Delta$ is a threshold value to control the sensitivity of $A$. The relationship between $A$ and $\Delta$ is shown in Figure 10. While $\Delta$ is too large, $A$ becomes slow in response to the metric changes and the performance degrades significantly. Hence, considering control of the sensitivity of $A$ by the parameters $\kappa$ and $\Delta$, the specific settings are adopted $\{\Delta=0.4, \kappa=10\}$ for the proposed path selection algorithm.

\section{Simulation Results and Analysis}

In this section, we validate the average queuing delay against the simulation results firstly. Then, we contrast the proposed path selection algorithm with the existing path selection algorithms. The proposed algorithm named "MASA" is the EASA based on the multipath Markov chain. Unless otherwise stated, the file/flow sizes are the exponential distribution, 


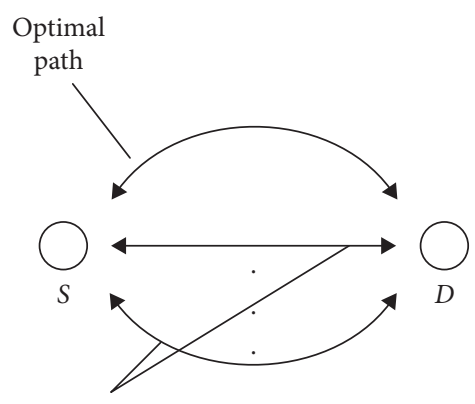

Other paths

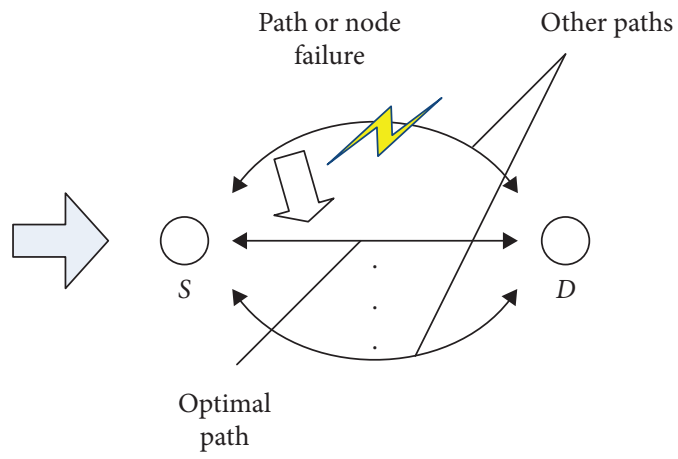

Figure 7: Multipath selection process.

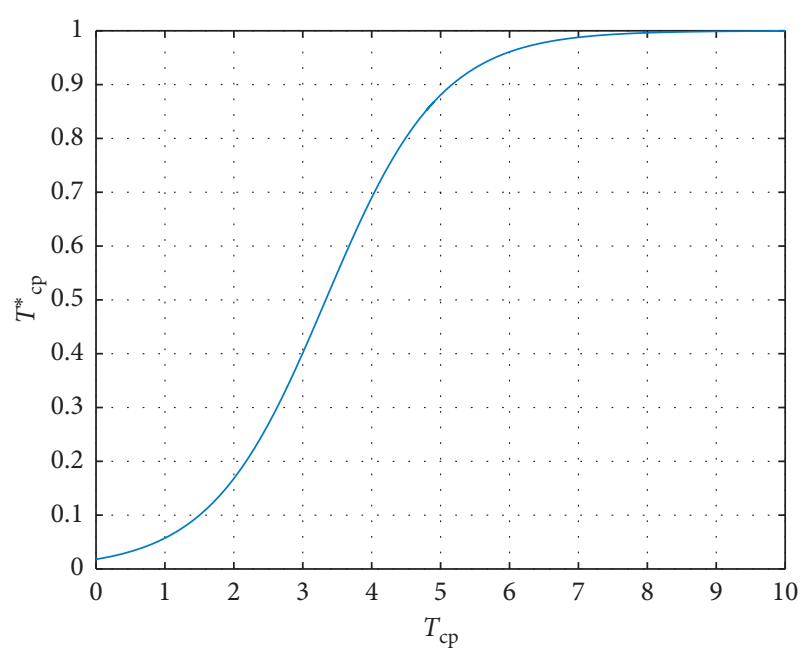

Figure 8: Relationship between $T_{\mathrm{CP}}$ and $T_{\mathrm{CP}}^{*}$.

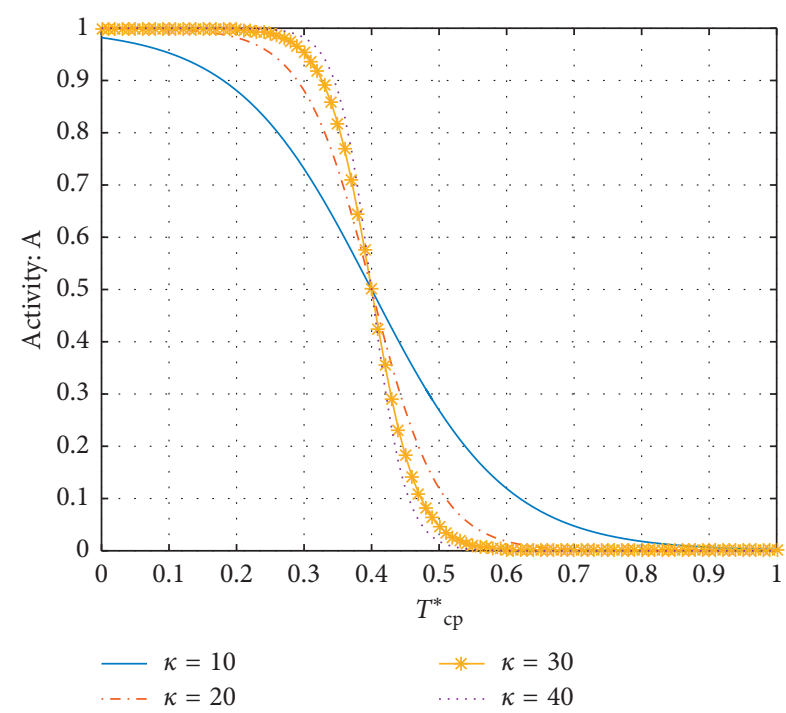

Figure 9: Different shapes of $A$ with different settings on $\kappa$.

and the file generation of users is a Poisson process and FCFS as the service policy.

5.1. Queuing Model Validation. We first validate the average queuing delay of the two-path Markov chain against the

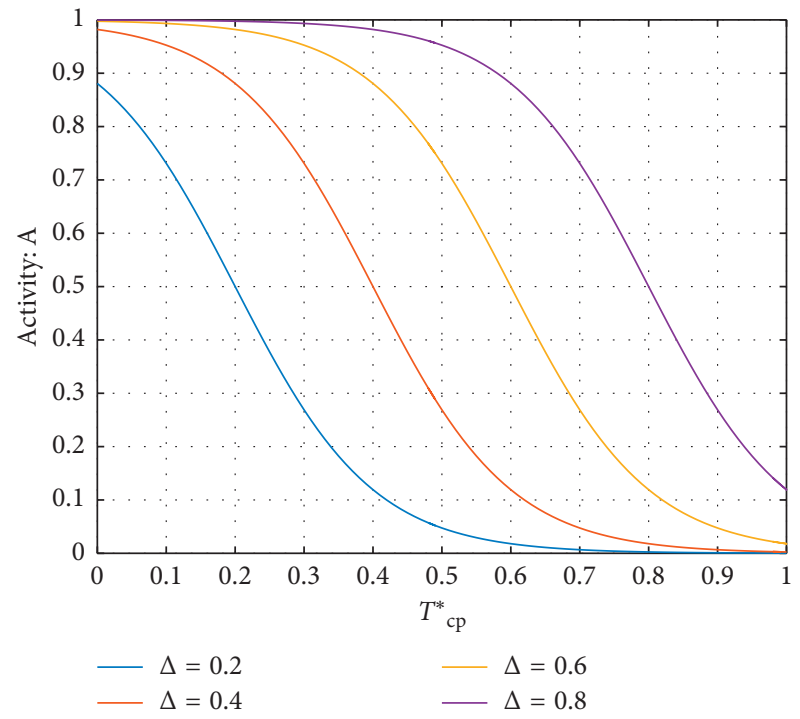

Figure 10: Different shapes of $A$ with different settings on $\Delta$.

simulations. The network topology of the two-path selection is shown in Figure 11. There are two nodes for the simulation, and each node accesses to the PLC or WN by PLC access point (PAP) or access point (AP), respectively. The data rate of the $\mathrm{WiFi}$ is assumed to be $2 \mathrm{Mbps}$ according to IEEE $802.11 \mathrm{~b}$. We assume that the data rate of the PLC is $1.5 \mathrm{Mbps}$ [36] and the mean packet size is $2 \mathrm{MB}$. Moreover, the arrival rate of the data transmitted between the two nodes is subject to the Poisson distribution. And the simulation parameters are shown in Table 2.

Figure 12 depicts the average queuing delay of the different arrival rates. The average queuing delay increases with the increase in the arrival rate, as expected, due to the queuing influence. It can be observed that there is a good match between the theories and the simulation results of the two networks. Moreover, it is can be seen that the average queuing delay curves grow faster while the arrival rate exceeds 1. This phenomenon indicates that the average queuing delay of the whole system model increases while the data grow lager enough. It is mainly caused by the lake of the bandwidth or the insufficient node processing speed. Therefore, we need the multipath model to provide the better transmission performance. 


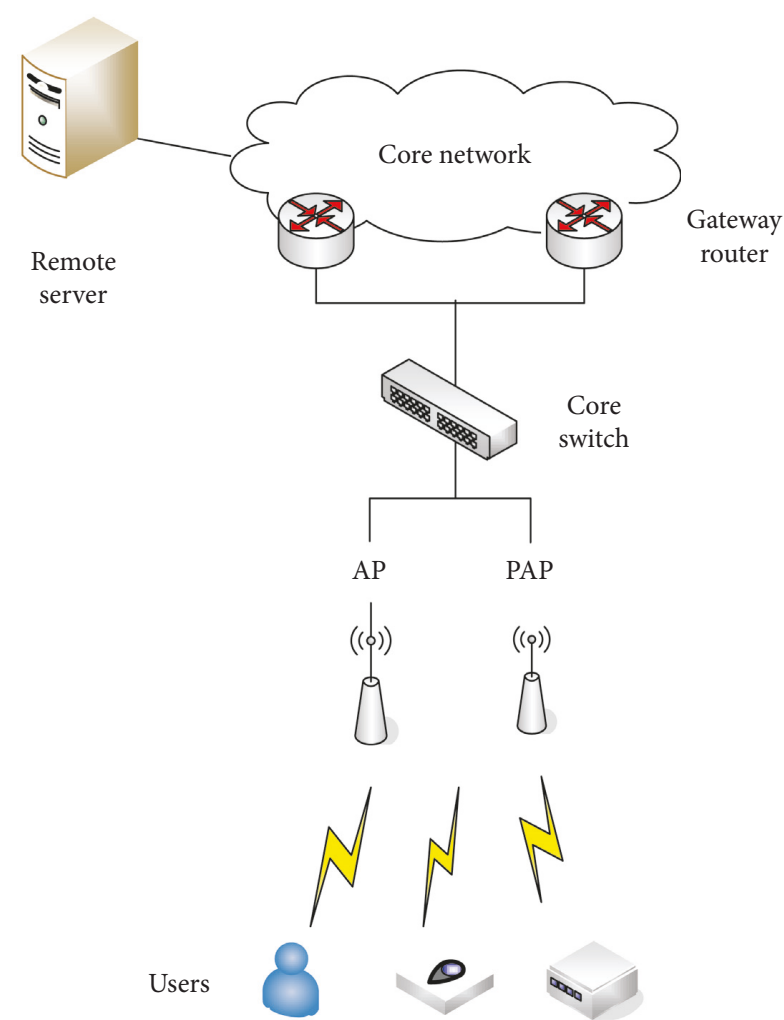

FIgURE 11: Network topology of the two-path selection.

TABLE 2: Variables and value.

\begin{tabular}{lc}
\hline Variable & Value \\
\hline$\mu_{1}$ & $1 / 150 \mathrm{~s}^{-1}$ \\
$\mu_{2}$ & $1 / 600 \mathrm{~s}^{-1}$ \\
$\eta_{1}$ & 0.5 \\
$\gamma_{1}$ & 0.5 \\
\hline
\end{tabular}

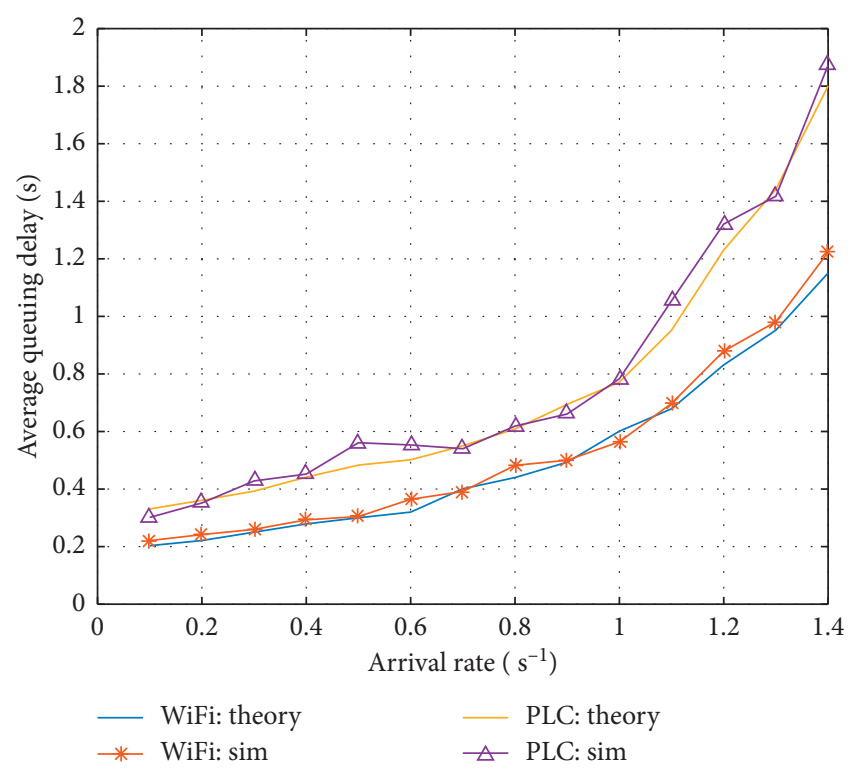

FIgURE 12: Average queuing delay of the different arrival rates.
Next, we consider the scenarios with the multiple access technologies (PLC, WiFi, LTE, cellular, and 3G) corresponding to the multipath model. The scenarios for different paths are shown in Table 3. $N$ is defined as the number of paths. Unless otherwise stated, the rates of data are given in Table 3 [31].

Figure 13 depicts the comparison of the average queuing delay between the two-path selection [16] and the multi-path selection to verify the performance improvement of the proposed algorithm. It is obvious that the average queuing delay of these results increases with the increased data and the average queuing delay in [16] is the biggest. Especially, the average queuing delay increases significantly, while the arrival rate exceeds 1 . Thus, it is can be seen that the selection algorithm in [16] is not suitable for the multipath scenario due to more bandwidth requirement with the increased data. Also, we can see that the average queuing delay in $N=3$ is larger than that in $N=4$ and $N=5$ due to the lack of bandwidth in $N=3$. However, the difference of the average queuing delay between $N=4$ and $N=5$ is not obvious. The reason is that more complex calculation is required with more complex Markov chain as shown in Figure 5. It is means that the time reduced by the more path selection is offset by the time consumed of the calculated complexity. Thus, the more the processing capacity the nodes need, the more the performance of the nodes decreases. In addition, due to the increased paths, the path selection becomes more frequent, leading to the decrease of the successful delivery rate of the data. Thus, the whole average queuing delay in $N=5$ does not have obvious advantage compared with that in $N=4$. And we focus on the path selection in $N=4$ for simplicity of research. The network topology is shown in Figure 14, which has 4 paths between the transmission nodes. Moreover, the users can access to the Internet through the different networks.

5.2. Multipath Model Validation. In [16], we only investigated the deliver delay of GA, AODV, and ASMC. Now, we give the results of the selection algorithm under the multipath scenario. And the influence of the nodes number on the average queuing delay, the delivery ratio, and the failure is also compared. Firstly, we compare the average queuing delay of the MASA, AODV, GA, SAW, and ISRO_PG as shown in Figure 15. AODV is a well-known reactive routing protocol which determines a unicast route to destinations with the advantage of the dynamic link conditions, low processing, and overhead [38]. GA can select the best choice available at every step without regard to the future consequences. This heuristic algorithm does not intend to find a best solution but terminates in a reasonable number of steps. The SAW, which is widely used in the recent research studies $[31,39]$, is one of the methods of MADM. And the ISRO_PG based on the game theory is also widely used in the recent research studies for the network selection problems $[40,41]$. The QoS requirements of the applications assumed in the SAW and ISRO_PG are referred to $[31,40]$, respectively. 
Table 3: Variables and definition.

\begin{tabular}{lcc}
\hline Number of paths & Type of paths & Rate (Mbps) \\
\hline \multirow{2}{*}{$N=3$} & PLC & 1.5 \\
& WiFi & 2 \\
& Cellular & 2 \\
\hline & PLC & 1.5 \\
$N=4$ & WiFi & 2 \\
& Cellular & 2 \\
\hline & LTE & $10[37]$ \\
& PLC & 1.5 \\
$N=5$ & WiFi1 & 2 \\
& Cellular & 2 \\
& LTE & 10 \\
\end{tabular}

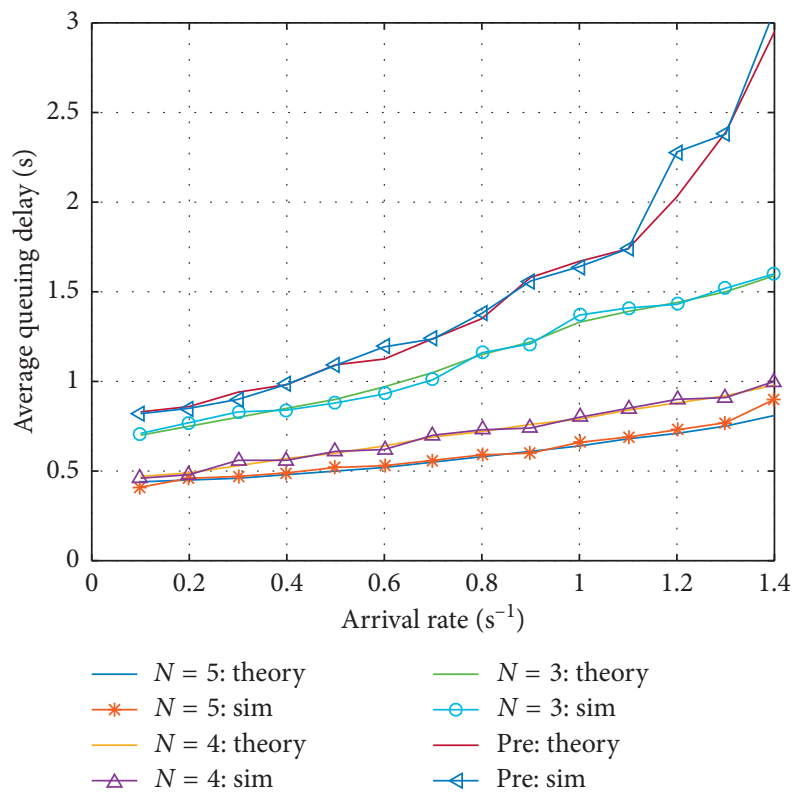

FIGURE 13: Different number paths against the average queuing delay.

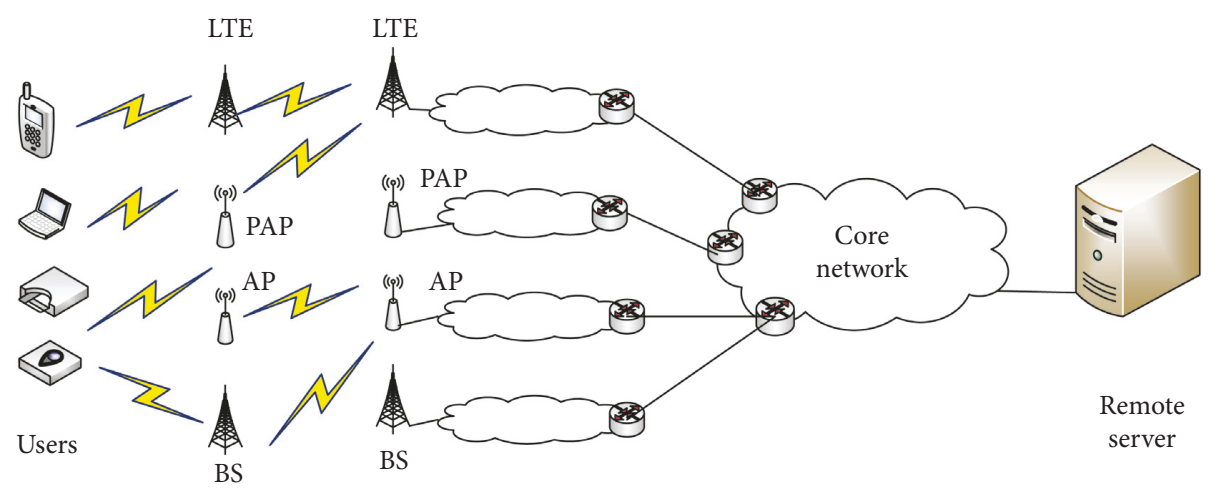

FIGURE 14: Network topology of multipath selection.

The average queuing delay comparisons between the MASA, AODV, GA, SAW, and ISRO_PG with the different number of nodes are illustrated in Figure 15. The abscissa is the number of nodes. While the number of nodes is less than 80 , it is not an obvious difference of the value between the average queuing delay in these algorithms. Then, with the increase in the number of nodes, the average queuing delay increases. While the number of nodes exceeds 80 , the differences of the average queuing delay between these algorithms become obvious. It can be seen that the MASA 


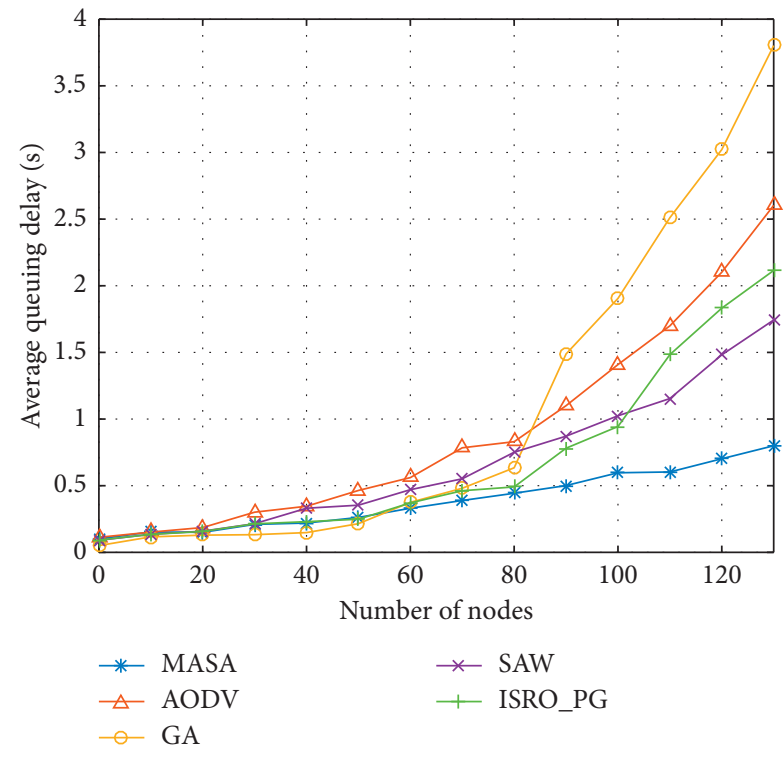

FIGURE 15: Average queuing delay against the number of nodes.

maintains low queuing delay and the GA has the maximum queuing delay. It is because the computations of the GA and AODV increase with the increase in the number of nodes. Since MASA has the capacity to allocate the path resources of the whole system, the average queuing delay of the MASA is the least of the compared algorithms. The ISRO_PG also allocates the network resources according to the environment. However, the computation times of the ISRO_PG increase with the increase in the number of nodes. Thus, the average queuing delay increases in the ISRO_PG scenario.

Secondly, we compare the transmission failure ratios of these algorithms with the different nodes number. Figure 16 shows the delivery ratio against the nodes number. As shown in this figure, the MASA achieves the higher delivery ratio than that of the AODV and SAW for the all cases. The GA provides the higher delivery ratio under the less dynamic conditions; however, the performance decreases gradually. Moreover, we can see that the performance of the GA decreases to the lowest in these algorithms while the number of occurrence exceeds 70. It is because that the GA introduces excessive overhead in the path maintenance and cannot update the path information promptly to adapt to the environment changes. Similarly, the delivery ratio of the ISRO_PG decreases with the increase of the failure nodes due to the less calculations.

Finally, we simulate the adaptability of these algorithms while the path is busy or the nodes are unable to work. Figure 17 also supports the superiority of MASA to the AODV, GA, SAW, and ISRO_PG in the same environment. As illustrated in Figure 17, there is not a huge difference of the delivery ratio between these algorithms while the number of nodes is small. Also, we can see that the deliver ratio of GA and AODV decreases with the increase in the invalid number of the nodes. In addition, the delivery ratio of the MASA maintains a steady state with the increasing failure nodes number. Its performance remains high against the increasing nodes. Since the MASA takes a probabilistic

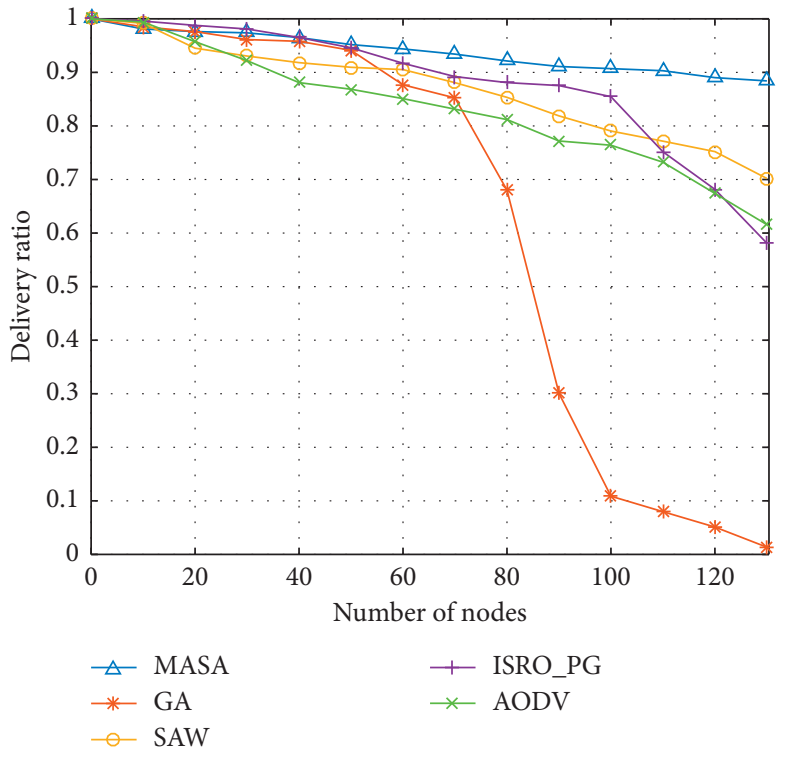

FIGURE 16: Delivery ratio against the number of nodes.

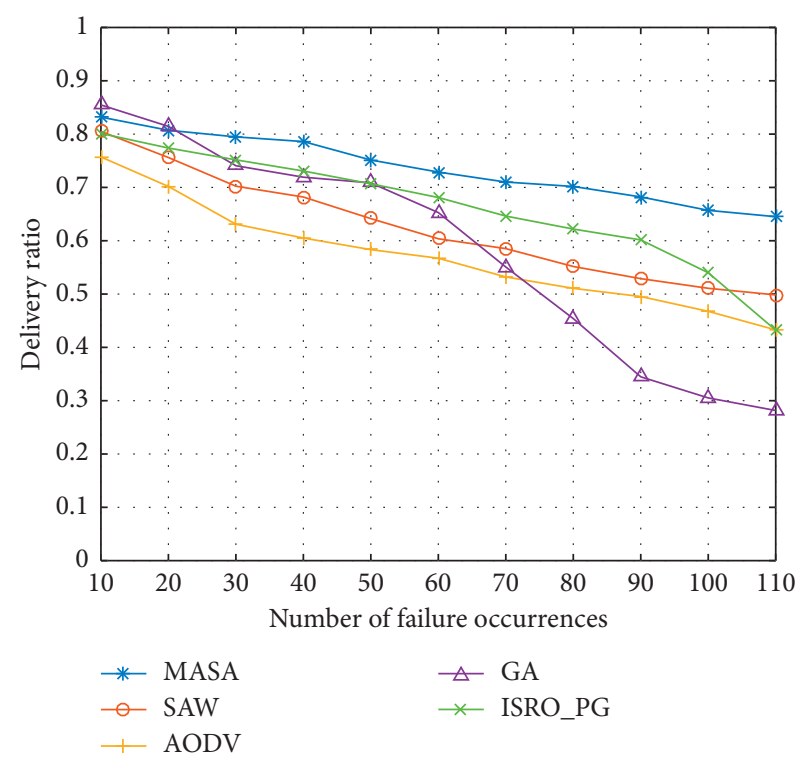

FIGURE 17: Delivery ratio against the failures.

approach of finding a good solution as the biological systems does, the data are allocated to the other path while CP is busy or not suitable for the work condition. The proposed algorithm improves the network robustness, improves the load balancing capacity and keeps the system stable.

\section{Conclusion}

In this paper, the adaptive HIoT model was proposed to solve the problems of the bandwidth and the spectrum shortage in WN. Firstly, we propose a novel HIoT which integrates the PLC and WN. There is a virtual layer in the HIoT, which provides unified interface to realize the uniform communication protocol. Then, we apply the MASA which is an algorithm-based Markov chain to allocate the 
network resources adaptively. The accuracy of evaluation results is analyzed by the mathematical calculations and simulations. Then, the validity and stability of the system model are verified in the different scenarios by the simulations. Finally, the investigations of delivery ratio and average queuing delay based on different nodes numbers, compared with the AODV, GA, SAW, and ISRO_PG, are discussed in the HIoT. The simulation results show that the algorithm in [16] is not suitable for the multipath selection with the increased data and the MASM has the network robustness, has the load balancing capacity, and keeps the system stable. In our future studies, we will focus on the priority of end users in the multipath HIoT model.

\section{Data Availability}

The simulation for multipath selection data used to support the findings of this study have been deposited in the Mendeley repository (https://doi.org/10.17632/z67jd9b3y5.2).

\section{Disclosure}

This work has been partially published in 2018 IEEE International Conference on Energy Internet (ICEI) [16] and extended in this work.

\section{Conflicts of Interest}

The authors declare that they have no conflicts of interest.

\section{Acknowledgments}

This work was supported by the National Science and Technology Major Project under Project 2018ZX03001016, the National Natural Science Foundation of China (61671073), the Project of Young Core Teacher in Higher Education of Anhui (gxyq2019070), and the Scientific Research Foundation of the Higher Education Institutions of Anhui Province, China (KJ2019A0630).

\section{References}

[1] A. Al-Fuqaha, M. Guizani, M. Mohammadi, M. Aledhari, and M. Ayyash, "Internet of things: a survey on enabling technologies, protocols, and applications," IEEE Communication Surveys Tutorials, vol. 17, no. 4, pp. 2347-2376, 2015.

[2] K. Ashton, "That "internet of things," thing," RFID Journal, vol. 22, pp. 97-114, 2009.

[3] D. Lopez-Perez, M. Ding, H. Claussen, and A. H. Jafari, "Toward $1 \mathrm{Gbps} / \mathrm{UE}$ in cellular systems: understanding ultradense small cell deployments," IEEE Communications Surveys Tutorials, vol. 17, no. 4, pp. 2078-2101, 2015.

[4] S. Kolipaka, B. N. Bhandari, and A. Dey, "Joint admission control and vertical handoff between WLAN and WIMAX in wireless mesh networks for QoS," in Proceedings of the 2016 IEEE International Conference on Engineering and Technology, pp. 1018-1023, IEEE, Coimbatore, India, March 2016.

[5] A. Gehlot and A. Rajavat, "Handoff between WiMAX and WiFi wireless networks," in Proceedings of the 2016 Symposium On Colossal Data Analysis and Networking, pp. 1-5, IEEE, Indore, India, March 2016.
[6] B. Angoma, M. Erradi, Y. Benkaouz, A. Berqia, and M. C. Akalay, "HaVe2W3G: a vertical handoff solution between WLAN, WiMAX and 3G networks," in Proceedings of the International Wireless Communications and Mobile Computing Conference, pp. 101-106, IEEE, Istanbul, Turkey, July 2011.

[7] V. Selis and A. Marshall, "A classification-based algorithm to detect forged embedded machines in IoT environments," IEEE Systems Journal, vol. 13, no. 1, pp. 389-399, 2019.

[8] S. A. Alharthi, P. Johnson, and M. A. Alharthi, "IoT architecture and routing for MV and LV smart grid," in Proceedings of the 2017 Saudi Arabia Smart Grid (SASG), pp. 1-6, IEEE, Jeddah, Saudi Arabia, December 2017.

[9] J.-S. Wu, S.-F. Yang, and B.-J. Hwang, "A terminal-controlled vertical handover decision scheme in IEEE 802.21-enabled heterogeneous wireless networks," International Journal of Communication Systems, vol. 22, no. 7, pp. 819-834, 2009.

[10] L. Hwang, P. Su, M. Zheng-Kun, M. Qing-min, and X. Minghai, "A simple additive weighting vertical handoff algorithm based on SINR and AHP for heterogeneous wireless networks," in Proceedings of the 2010 International Conference on Intelligent Computation Technology and Automation, vol. 1, pp. 347-350, IEEE, Changsha, China, May 2010.

[11] E. Lee, C. Choi, and P. Kim, "Intelligent handover scheme for drone using fuzzy inference systems," IEEE Access, vol. 5, pp. 13712-13719, 2017.

[12] S. Kunarak and R. Suleesathira, "Predictive RRS with fuzzy logic based vertical handoff algorithm in heterogeneous wireless networks," in Proceedings of the 2010 10th International Symposium On Communications and Information Technologies, pp. 26-29, IEEE, Ho Chi Minh City, Vietnam, October 2010.

[13] D. Fudenberg and J. Tirole, Game Theory, MIT, Cambridge, MA, USA, 1991.

[14] I. Malanchini, M. Cesana, and N. Gatti, "Network selection and resource allocation games for wireless access networks," IEEE Transactions on Mobile Computing, vol. 12, no. 12, pp. 2427-2440, 2013.

[15] M.-A. Koulali, S. Koulali, H. Tembine, and A. Kobbane, "Industrial internet of things-based prognostic health management: a mean-field stochastic game approach," IEEE Access, vol. 6, pp. 54388-54395, 2018.

[16] H. Wu, X. Wen, Z. Lu, and S. Huang, "Markov chain based on path selection in the heterogeneous internet of things model," in Proceedings of the IEEE International conference on Energy Internet (ICEI), May 2018.

[17] M. R. Palattella, N. Accettura, X. Vilajosana et al., "Standardized protocol stack for the Internet of (important) things," IEEE Communications Surveys \& Tutorials, vol. 15, no. 3, pp. 1389-1406, 2013.

[18] J. W. Hui, A. Terzis, S. Dawson-Haggerty, D. E. Culler, J. W. Hui, and P. Levis, "Connecting low power and lossy network to the Internet," IEEE Communications Magazine, vol. 49 , no. 4 , pp. 96-101, 2011.

[19] M. Segal, "A multiserver system with preemptive priorities," Operations Research, vol. 18, no. 2, pp. 316-323, 1970.

[20] N. TianandZ and G. Zhang, Vacation Queuing Models: Theory and Applications, Springer, New York, NY, USA, 2006.

[21] N. Perel and U. Yechiali, "Queues with slow servers and impatient customers," European Journal of Operational Research, vol. 201, no. 1, pp. 247-258, 2010.

[22] S. Floyd and V. Jacobson, "Random early detection gateways for congestion avoidance," IEEE/ACM Transactions on Networking, vol. 1, no. 4, pp. 397-413, 1993. 
[23] W. Wang, C. Yin, G. Yan, and B.-H. Wang, "Integrating local static and dynamic information for routing traffic," Physical Review E, vol. 74, no. 1, Article ID 016101, 2006.

[24] J. J. Zhang, W. L. Xu, and L. G. Wang, “An improved active queue management algorithm based on nonlinear smoothing," Advanced Materials Research, vol. 295-297, pp. 18231828, 2011.

[25] U. Yechiali and P. Naor, "Queuing problems with heterogeneous arrivals and service," Operations Research, vol. 19, no. 3, pp. 722-734, 1971.

[26] S. M. Ross, Stochastic Processes, John Wiley and Sons, Hoboken, NJ, USA, 2nd edition, 1996.

[27] J. L. Gustafson, Encyclopedia of Parallel Computing, Springer, Boston, MA, USA, 2011.

[28] I. L. Mitrany and B. Avi-Itzhak, "A many-server queue with service interruptions," Operations Research, vol. 16, no. 3, pp. 628-638, 1968 .

[29] K. Leibnitz, N. Wakamiya, and M. Murata, "Biologically inspired self-adaptive multi-path routing in overlay networks," Communications of the ACM, vol. 49, no. 3, pp. 62-67, 2006.

[30] K. Leibnitz and M. Murata, "Attractor selection and perturbation for robust networks in fluctuating environments," IEEE Network, vol. 24, no. 3, pp. 14-18, 2010.

[31] S. Kajioka, N. Wakamiya, and M. Murata, "Autonomous and adaptive resource allocation among multiple nodes and multiple applications in heterogeneous wireless networks," Journal of Computer and System Sciences, vol. 78, no. 6, pp. 1673-1685, 2012.

[32] D. Tian, J. Zhou, Y. Wang, Y. Lu, H. Xia, and Z. Yi, "dynamic and self-adaptive network selection method for multimode communications in heterogeneous vehicular telematics," IEEE Transactions on Intelligent Transportation Systems, vol. 16, no. 6, pp. 3033-3049, 2015.

[33] Z. Hu, X. Wen, Z. Lu, and W. Jing, “AORS: adaptive mobile data offloading based on attractor selection in heterogeneous wireless networks," Wireless Networks, vol. 23, no. 3, pp. 831-842, 2017.

[34] Y. Li, J. Wang, and Y. Xu, “Adaptive intentional connection rerouting for traffic grooming in WDN mesh networks with biological attractor selection," in Proceedings of the 2010 16th Asia-Pacific Conference On Communications, pp. 83-88, IEEE, Auckland, New Zealand, November 2010.

[35] H. Wu, X. Wen, Z. Lu, and Q. Pan, "Mobile data offloading under attractor selection in heterogeneous networks," in Proceedings of the 2017 International Symposium on Wireless Communication Systems (ISWCS), pp. 164-169, IEEE, Bologna, Italy, August 2017.

[36] W. Liu, H. Widmer, and P. Raffin, "Broadband PLC access systems and field deployment in European powerline networks," IEEE Communications Magazine, vol. 41, no. 5, pp. 114-118, 2003.

[37] S. Chen, J. Hu, Y. Shi, and L. Zhao, "LTE-V: a TD-LTE-based V2X solution for future vehicular network," IEEE Internet of Things Journal, vol. 3, no. 6, pp. 997-1005, 2016.

[38] J. J. Ferronato and M. A. S. Trentin, "Analysis of routing routing protocols OLSR, AODV and ZRP in real urban vehicular scenario with density variation," IEEE Latin America Transactions, vol. 15, no. 9, pp. 1727-1734, 2017.

[39] H. Yu, Y. Ma, and J. Yu, "Network selection algorithm for multiservice multimode terminals in heterogeneous wireless networks," IEEE Access, vol. 7, pp. 46240-46260, 2019.

[40] J. Zhao, Research on multi-resources optimization strategies in heterogeneous wireless networks, Beijing University of Posts and Telecommunications, Beijing, China, Ph.D. dissertation, 2015.

[41] T. Zhong, T. Peng, and Y. Zhang, "A game theory scheme combined with the interference region in cellular D2D heterogeneous networks," in Proceedings of the 2018 IEEE/CIC International Conference on Communications in China (ICCC Workshops), pp. 167-171, IEEE, Beijing, China, August 2018. 


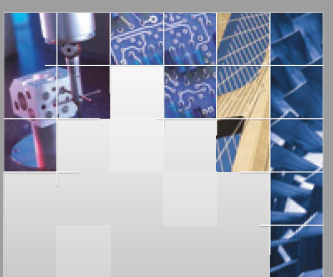

\section{Enfincering}
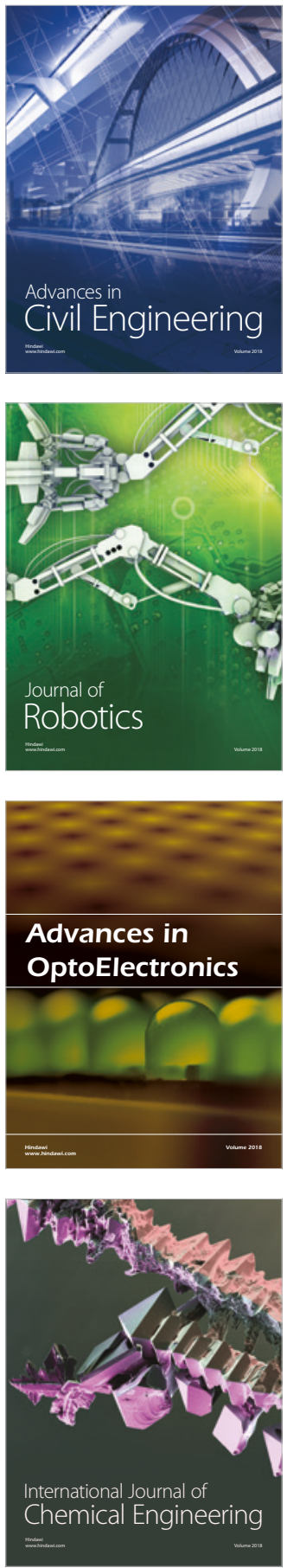

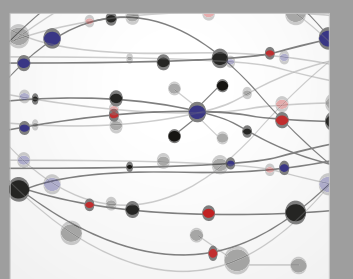

\section{Rotating \\ Machinery}

The Scientific World Journal

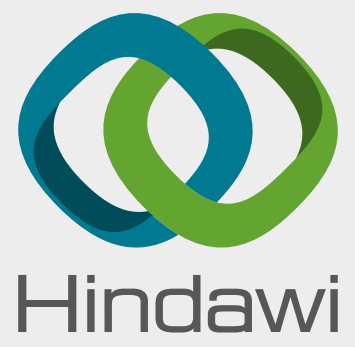

Submit your manuscripts at

www.hindawi.com
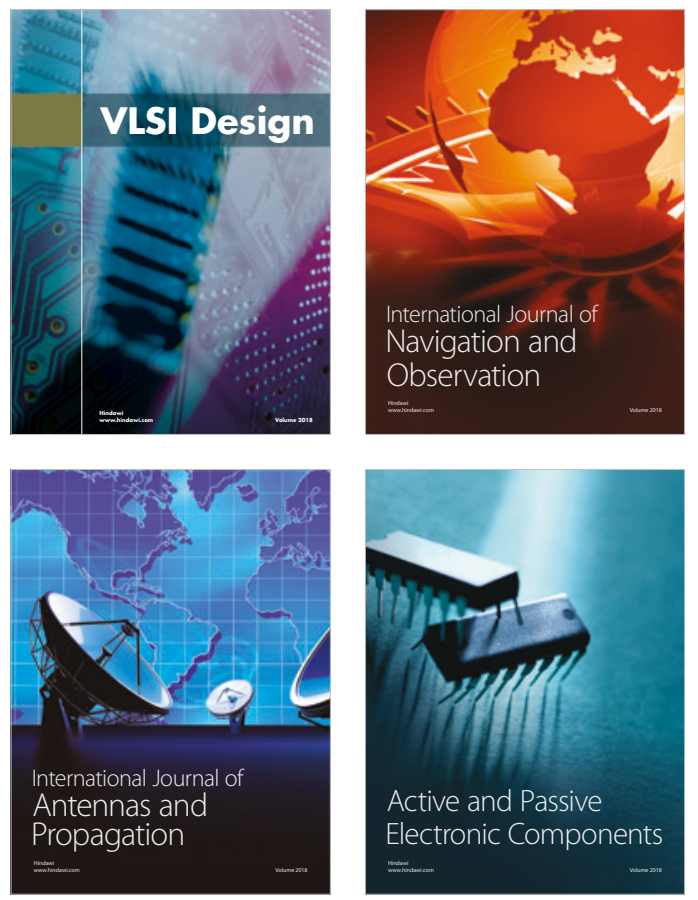
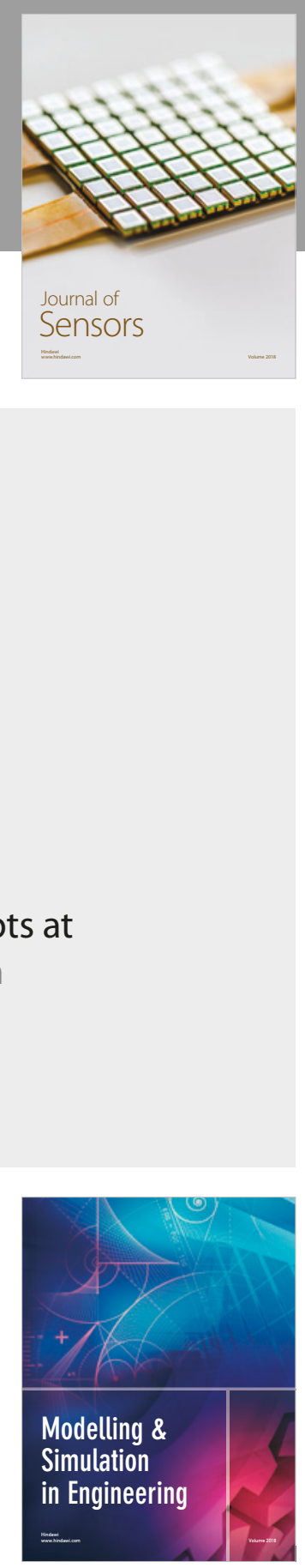

\section{Advances \\ Multimedia}
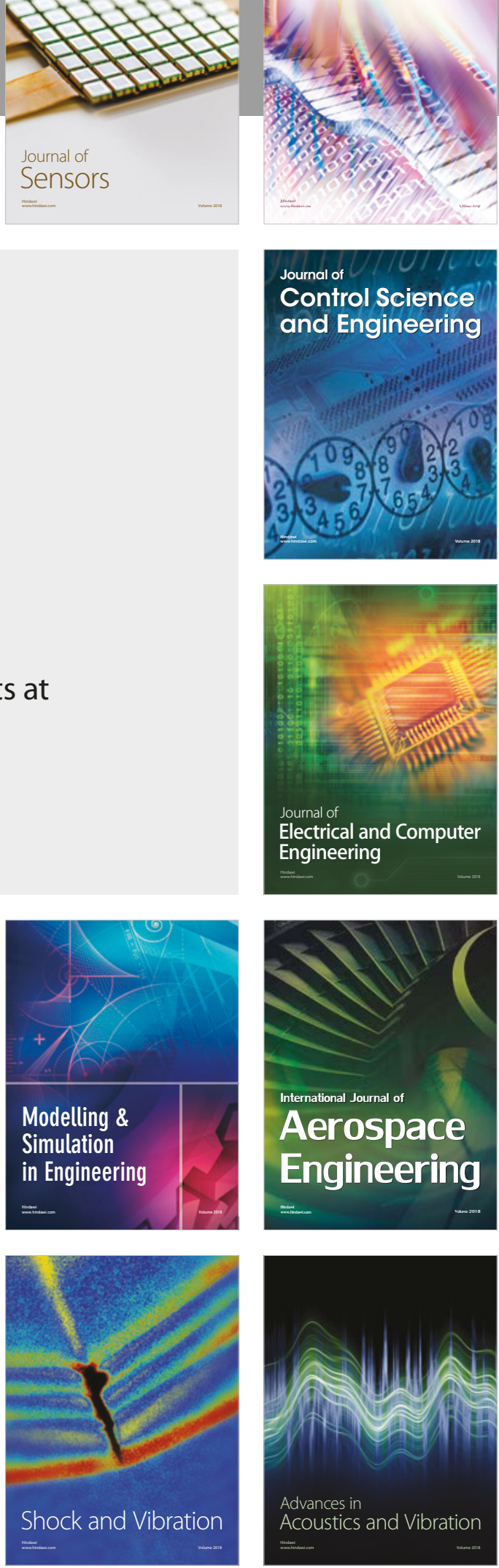